\title{
Edge corrections for parallel-plate capacitors $\dagger$
}

\author{
EHUD YARIV \\ Department of Mathematics, Technion -Israel Institute of Technology, Haifa 32000, Israel \\ email:udi@technion.ac.il
}

(Received 28 June 2019; revised 25 February 2020; accepted 20 March 2020; first published online 30 April 2020)

\begin{abstract}
As a result of field fringing, the capacitance of a parallel-plate capacitor differs from that predicted by the textbook formula. Using singular perturbations and conformal mapping techniques, we calculate the leading-order correction to the capacitance in the limit of large aspect ratio. We additionally obtain a comparable approximation for the electrostatic attraction between the plates.
\end{abstract}

Key words: Singular perturbations, conformal mappings

2020 Mathematics Subject Classification: Primary: 35B25; 35Q60; 41A60; 31A99. Secondary: $42 \mathrm{~B} 37$

\section{Introduction}

The calculation of the capacitance of a parallel-plate condenser appears in any electrostatics textbook. Assuming that the field lines are (i) confined between the plates and (ii) perpendicular to them, one readily finds from Gauss law that the capacitance per unit area is given by the ratio of the vacuum permittivity $\varepsilon_{0}$ to the distance between the plates. In the convenient two-dimensional idealisation, involving two plates of length $2 l$ and separation $2 h$, the capacitance per unit length is given by $\varepsilon_{0} \lambda, \lambda=l / h$ being the aspect ratio.

Of course, for any finite value of $\lambda$, however, large, the field lines are not confined between the plates; in the vicinity of the edges, moreover, they are clearly not perpendicular to the plates. There is accordingly an interest in correcting the 'idealised-capacitor' formula. Assuming plates of zero thickness, Thomson [30] suggested the following approximation to the capacitance (per unit length), normalised by $\varepsilon_{0}$,

$$
\lambda+\frac{\ln (\pi \lambda)+1}{\pi}+\cdots
$$

Approximation (1.1) incorporates the relative asymptotic corrections of both $O(1)$ and $O(\ln \lambda)$. When considering $O(\ln \lambda)$ terms on par with $O(1)$ terms, as is the convention in asymptotic analysis [12], this approximation appears to effectively provide a leading-order edge correction.

In the literature, Thomson's approximation is typically derived in a heuristic manner, which is forced by the appearance of a divergent integral (see, e.g., the treatment in Section 8.2.3 of Binns and Lawrenson [3]). Edge corrections are briefly discussed by Landau and Lifshitz [21,

$\dagger$ This work was supported by the Israel Science Foundation (Grant no. 1081/16). 
Section 3], where the authors explain that, due to their intuitive handling of a logarithmically divergent integral, only the $O(\ln \lambda)$ correction term can be trusted and that the calculation of the associated $O(1)$ correction term 'demands considerably more elaborate methods'.

It turns out that the above reservation by Landau and Lifshitz applies to Thomson's approximation. The validity of that approximation was put into doubt by the expression obtained by Bromwich [4] in 1902,

$$
\lambda+\frac{\ln (2 \pi \lambda)+1}{\pi}+\cdots,
$$

which differs from (1.1) at $O(1)$. In 1924, Love [23] obtained an exact solution (valid for arbitrary values of $\lambda$ ) of the two-dimensional capacitance problem. This solution is expressed in terms of elliptic functions and is accordingly rather cumbersome. Nonetheless, when degenerated to the case of large aspect ratio, it reduces to (1.2). Thomson's approximation indeed misses a term.

A comparable, though less dramatic, discrepancy appeared in the related problem of disk capacitors, for which a non-rigorous approximation was originally provided by Kirchhoff [19]. A later approximation by Ignatowsky [14] differed from that of Kirchhoff at the $O(1 / \lambda)$ correction. In this problem, the exact solution of Sneddon [29, Chapter 8] revealed that it is the Kirchhoff approximation which is the correct one.

As an alternative to asymptotic limits of exact solutions, large-aspect-ratio approximations may be systematically obtained using asymptotic methods. There are essentially two manners by which such approximations are derived in that context. The first makes use of an integral representation of the potential problem, with the pertinent integral equations being solved in the appropriate asymptotic limit [1]. This approach has been used extensively in the analysis of disk capacitors [7, 13,22] as well as in the related problem of added mass calculation [2]. The second approach, which constitutes the natural follow-up of the intuitive calculations of Kirchhoff and Thomson, attempts to solve Laplace's equation directly, with the high-aspect-ratio singularity being addressed from the outset using singular perturbations. This direct approach was carried out by Shaw [28] for both strip and disk capacitors.

While the correct high-aspect-ratio approximations are available in the literature, they are still largely unfamiliar. It is possible to find papers in the engineering community that, being apparently unaware of the Bromwich-Love approximation (1.2), use Thomson's erroneous approximation (1.1) as a benchmark against which numerical calculations are compared [25]. There are several reasons for this unfamiliarity. Traditionally, the strip-capacitor problem was viewed as an intermediate step in the analysis of a disk capacitor. Indeed, the BromwichLove approximation (1.2) has been reproduced in several of the above-mentioned disk-capacitor analyses, see, for example, equation (3.12) in Hutson [13] and equation (4.13) in Chew and Kong [7]. As these analyses are quite involved, they are only known to a small group of experts. Furthermore, in the few cases where the two-dimensional geometry was directly addressed, this was done in a rather general context which has rendered the analysis quite technical. Thus, Shaw [28] considered plates of a finite thickness, while Chew and Kong [7] allowed for a dielectric slab between the plates. Another possible reason for the unfamiliarity has to do with the appearance of key relevant papers [28] in the fluid-mechanical literature. This has to do with the (virtually unknown) analogy between the electrostatic problem and the problem of inertialess flow in a parallel-plate viscometer [8] (which implies, in turn, an analogy between the capacitance in the electrostatic problem and the torque in the hydrodynamic problem). 


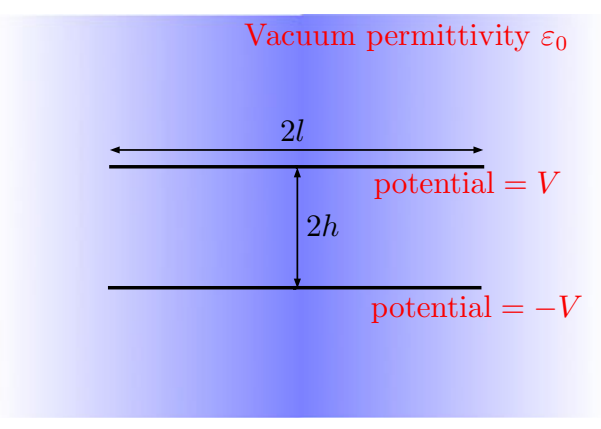

FIGURE 1. Parallel-plate geometry.

It therefore appears desirable to present a systematic derivation of the Bromwich-Love approximation (1.2), which applies in the simplest problem of zero-thickness plates. The scope of such a goal is more modest than that achieved by Shaw [28], which has actually gone to higher asymptotic orders. However, Shaw's work is very general and not easy to read. The restricted derivation which follows may accordingly serve as a convenient introduction for non-experts, supplementing both fundamental analyses of capacitance calculations $[15,16,32]$ as well as less mathematical discussions of the fringing effect $[6,10,20,24,26]$. This educational goal will be accomplished in the present paper using matched asymptotic expansions [12], where the electric potential is solved in different subdomains (defined via appropriate limit processes). The capacitance may then be obtained by carefully adding the contributions from the respective regions, with the result being independent of the exact manner by which these regions are delineated. The asymptotic solution of the electric potential will also be used to obtain an approximation for the attractive force between the plates; since the mathematical analog of that force has no physical meaning in the problem of parallel-plate viscometer, this quantity has not been sought by Shaw [28].

As will become evident, the major task in the present work involves the calculation of the electric potential near the 'edge' of the capacitor. This task is accomplished using conformal mapping techniques. Consistently with the expository nature of this paper, two alternative mappings will be described. Similarly, the electrostatic attraction is determined using two different approaches, one direct, using the Maxwell stress concept, and one indirect, using the principle of virtual work.

\section{Formulation}

Consider a two-dimensional capacitor, made out of two thin conducting plates of length $2 l$. The plates are parallel to each other and separated at a distance $2 h$ apart (see Figure 1). The plates are set at electric potentials $\pm V$ relative to the potential at infinity. Our goal is the capacitance, defined by

$$
C=\frac{Q}{2 V},
$$

in which, $Q$ is the charge on the positive plate. Since $Q$ is linear in $V$, the capacitance is independent of it. 


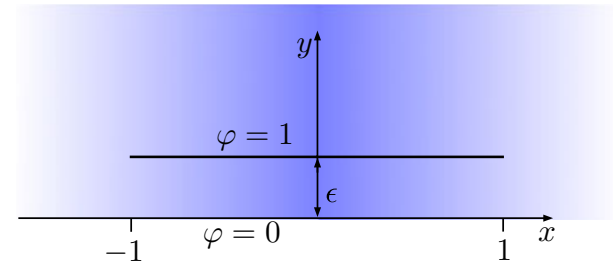

FIGURE 2. Dimensionless problem in the upper $x y$-plane.

Normalising length variables by $l$, we employ Cartesian $x y z$ coordinates with the $x$-axis running parallel to the plates, which extend between $x=-1$ and $x=1$, and the $y$-axis running perpendicular to them. We employ a dimensionless notation where electric potentials are normalised by $V$ and charges (per unit length in the $z$-direction) are normalised by $\varepsilon_{0} V$, $\varepsilon_{0}$ being the permittivity of vacuum; the capacitance (per unit length in the $z$-direction) is consistently normalised by $\varepsilon_{0}$. In what follows, we find it preferable to employ $\epsilon=h / l$ instead of the aspect ratio $\lambda$.

The electric potential $\varphi$ is governed by (i) Laplace's equation,

$$
\frac{\partial^{2} \varphi}{\partial x^{2}}+\frac{\partial^{2} \varphi}{\partial y^{2}}=0 ;
$$

(ii) the Dirichlet condition $\varphi= \pm 1$ at the two plates (respectively, positioned at $y= \pm \epsilon$ ); and (iii) far-field decay,

$$
\varphi=O(1 / r) \quad \text { for } \quad r=\sqrt{x^{2}+y^{2}} \ll 1,
$$

the latter condition following from the absence of net charge in the two-plate system. It is evident from the problem formulation that $\varphi$ is an even function of $x$ and an odd function of $y$. We hereafter consider only $y>0$, adding the symmetry condition (see Figure 2)

$$
\varphi=0 \quad \text { at } \quad y=0 .
$$

Since the dimensionless potential difference between the plates is 2 , the dimensionless capacitance is given by $c=q / 2$, wherein $q$ is dimensionless charge on the upper plate (cf. (2.1)). Making use of the two-dimensional variant of Gauss law, we find that

$$
q=-\oint \nabla \varphi \cdot \hat{\mathbf{n}} d s
$$

where the line integral may be carried out over any simple closed contour which encircles (only) the upper plate, $\hat{\mathbf{n}}$ denotes an outward-pointing unit vector normal to that contour and $d s$ is a differential length element. Upon deforming the contour and making use of the far-field decay (2.3), we represent $c$ as an integral over the $x$-axis, namely

$$
c=\left.\frac{1}{2} \int_{-\infty}^{\infty} \frac{\partial \varphi}{\partial y}\right|_{y=0} d x,
$$

or, upon making use of the symmetry about $x=0$,

$$
c=\left.\int_{0}^{\infty} \frac{\partial \varphi}{\partial y}\right|_{y=0} d x .
$$




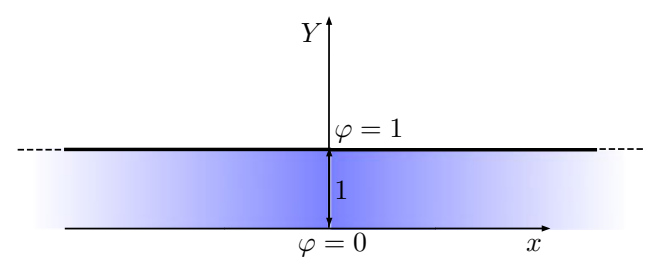

FIGURE 3. The gap region.

The dimensionless capacitance $c$ is a function of $\epsilon$, the single parameter in the problem. Our goal is to derive an approximation in the small-gap limit, $\epsilon \ll 1$. When the fringing effect is ignored and one consider only the region between the plates, it is evident that, in that region,

$$
\varphi \approx y / \epsilon
$$

Substitution into (2.7) then gives

$$
c \approx 1 / \epsilon
$$

How is this 'idealised-capacitor' value affected by the finite aspect ratio?

\section{Asymptotic regions}

As $\epsilon \rightarrow 0$, it is convenient to distinguish between three 'asymptotic regions'. The 'outer region' corresponds to $x$ and $y$ being $O(1)$. The 'gap region' corresponds to $x \in(-1,1)$ being $O(1)$ but $y=O(\epsilon)$. The 'edge region', at $O(\epsilon)$ distances from the edge $(1,0)$, is where both $x-1$ and $y$ are $O(\epsilon)$. (By symmetry, another such region is formed about $(-1,0)$.) To represent the conceptual decomposition of the problem domain into these region, we define the stretched coordinates

$$
\begin{aligned}
& X=\frac{x-1}{\epsilon}, \\
& Y=\frac{y}{\epsilon} .
\end{aligned}
$$

The different regions then correspond to the manner by which the limit $\epsilon \rightarrow 0$ is attained. In the outer region, $x$ and $y$ are held fixed as $\epsilon \rightarrow 0$; in the gap region, $x$ and $Y$ are held fixed (with $x \in(-1,1))$; in the edge region, $X$ and $Y$ are held fixed.

\section{Leading-order electric potential}

We now derive the leading-order approximations to the electric potentials in the three respective regions, noting that the far-field condition (2.3) applies only in the outer region. In the leadingorder gap region problem, the ends are removed to infinity. Laplace's equation (4.10) becomes

$$
\frac{\partial^{2} \varphi}{\partial Y^{2}}+\epsilon^{2} \frac{\partial^{2} \varphi}{\partial x^{2}}=0
$$




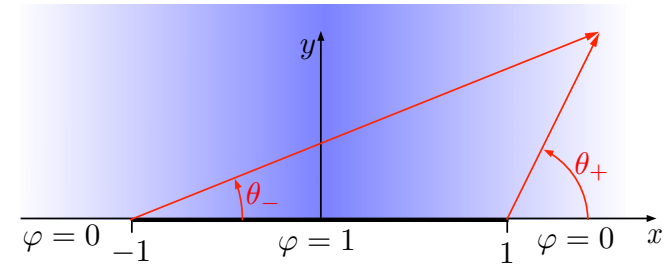

FIGURE 4. Outer region.

A solution that satisfies the boundary conditions (see Figure 3 ) is

$$
\varphi \sim Y
$$

this, of course, is the dimensionless version of (2.8). It may appear that (4.2) is only a leadingorder solution; recalling, however, the form of the eigenfunctions of Laplace's equation in the gap geometry, it is evident that the asymptotic correction to (4.2) is exponentially small [9].

In the outer region, at leading order, one has the discontinuous Dirichlet condition at $y=0$ (see Figure 4):

$$
\varphi= \begin{cases}0 & |x|>1 \\ 1 & |x|<1 .\end{cases}
$$

Recalling that a polar angle is a solution of Laplace's equation (4.10), we claim that

$$
\varphi \sim \frac{1}{\pi}\left(\theta_{+}-\theta_{-}\right)
$$

wherein the polar angles $\theta_{ \pm}$are indicated in Figure 4. Indeed, it is readily seen that (4.4) satisfies both (2.3) and (4.3). Expressing the angles in terms of the $x y$ coordinates, we alternatively have

$$
\varphi \sim \frac{1}{\pi}\left(\arctan \frac{y}{x-1}-\arctan \frac{y}{x+1}\right),
$$

where it is hereafter understood that the arctangent is between 0 and $\pi$.

\subsection{Edge region}

The only region where the calculation of $\varphi$ requires some effort is the edge. Defining $Z=X+i Y$, it is here convenient to employ a conformal mapping $Z=f(\zeta)$ from the complex $\zeta$-plane to the upper-half complex $Z$-plane. In particular, the degenerate polygon geometry of the edge suggests the use of a Schwarz-Christoffel mapping [5] from the upper-half $\zeta$-plane (see Figure 5). Upon setting the critical points of the transformation in the manner described in Figure 5, the mapping $f$ must satisfy

$$
f^{\prime}(\zeta)=M \frac{\zeta+1}{\zeta}
$$

Integration thus yields

$$
f(\zeta)=M(\zeta+\log \zeta)+N
$$

where the principal branch of the logarithm is taken. 

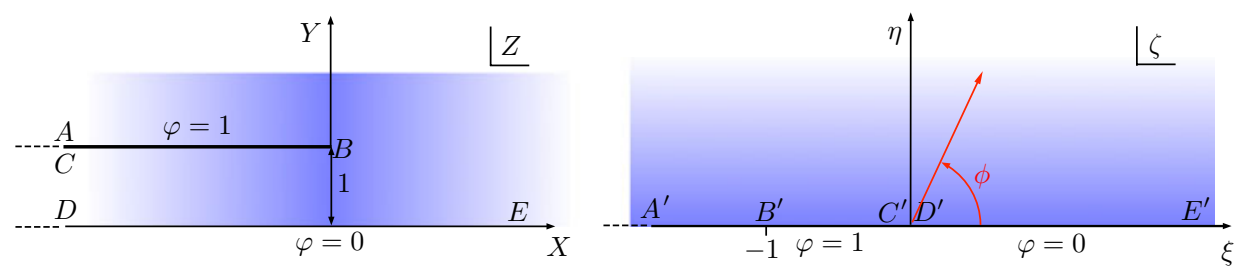

FIGURE 5. Mapping to the edge region.

The coefficient $M$ is determined by the requirement that the semi-width of the capacitor in the $X Y$-plane is unity. In terms of $f$, this requirement reads

$$
\lim _{\rho \searrow 0}[f(-\rho)-f(\rho)]=i .
$$

Making use of (4.7), we then obtain $M=1 / \pi$. To determine $N$, we impose the condition $f(-1)=$ $i$ (see Figure 5), which gives $N=1 / \pi$. We conclude that

$$
f(\zeta)=\frac{\zeta+\log \zeta+1}{\pi}
$$

In what follows, we write $\zeta=\xi+i \eta$. Since Laplace's equation is conformally invariant [5], we need to solve

$$
\frac{\partial^{2} \varphi}{\partial \xi^{2}}+\frac{\partial^{2} \varphi}{\partial \eta^{2}}=0 \quad \text { for } \quad \eta>0
$$

As Dirichlet conditions are also conformally invariant [5], the following applies at $\eta=0$ :

$$
\varphi= \begin{cases}1 & \xi<0 \\ 0 & \xi>0\end{cases}
$$

The solution of (4.10)-(4.11) is simply

$$
\varphi=\frac{\phi}{\pi}
$$

wherein

$$
\phi=\arctan \frac{\eta}{\xi}
$$

is the polar angle in the $\xi \eta$-plane (see Figure 5).

Note that the real and imaginary parts of (4.7), respectively, give

$$
\begin{aligned}
& X=\frac{\xi+\ln |\xi|+1}{\pi}, \\
& Y=\frac{\eta+\phi}{\pi} .
\end{aligned}
$$



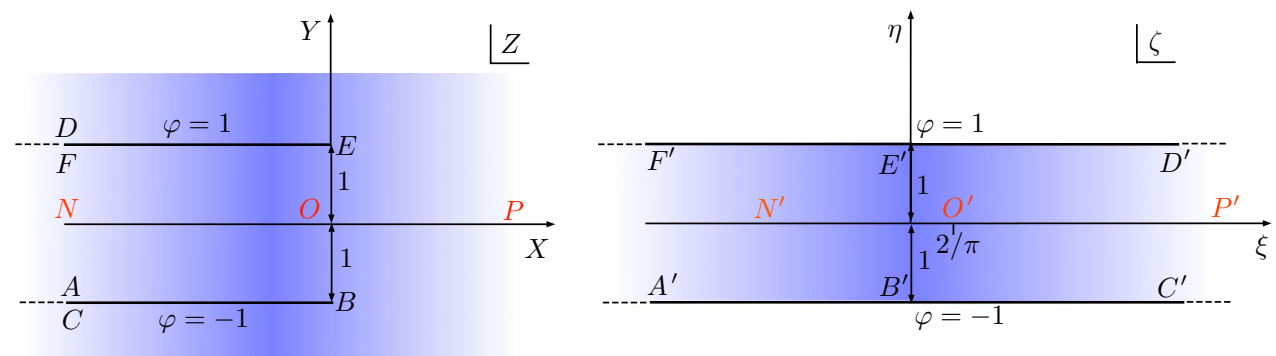

FIGURE 6. Alternative mapping to the edge region.

When reaching out to the outer region, $\xi$ and $\eta$ become large (see Figure 5). Relations (4.14) then give

$$
\begin{aligned}
& X \sim \frac{\xi}{\pi}, \\
& Y \sim \frac{\eta}{\pi},
\end{aligned}
$$

where the relative error is algebraically small (i.e. smaller than some positive power of $|\zeta|^{-1}$ ). At leading order, we then obtain from (4.13) $\phi \sim \arctan (Y / X)$. Reverting to the unscaled coordinates using (3.2b) and substituting into (4.12) give

$$
\varphi \sim \frac{1}{\pi} \arctan \frac{y}{x-1} .
$$

Thus, asymptotic matching with the outer potential (4.5) is trivially satisfied. Similarly, when approaching the gap region, $\xi$ and $\eta$ are small (see Figure 5), so relations (4.14) become

$$
\begin{aligned}
& X \sim \frac{\ln |\xi|+1}{\pi}, \\
& Y \sim \frac{\phi}{\pi},
\end{aligned}
$$

with an algebraically small relative error. From (4.12) and (4.17b), we find that $\varphi \sim Y$, so that asymptotic matching with the gap solution (4.2) is, too, trivially satisfied.

\subsection{Edge region: an alternative mapping}

Instead of the Schwarz-Christoffel transformation (4.9), we can employ the transformation [31]

$$
f(\zeta)=\zeta+\frac{1}{\pi}\left(e^{\pi \zeta}+1\right)
$$

which maps the strip $|\eta| \leq 1$ to the entire $Z$-plane. The manner by which it maps the edge region is described in Figure 6. Notably, the $\xi$-axis is mapped onto the $X$-axis.

We accordingly need again to solve Laplace's equation (4.10), but now with conditions (4.11) replaced by

$$
\varphi= \pm 1 \quad \text { at } \quad \eta= \pm 1
$$


The solution is simply (cf. (4.12))

$$
\varphi=\eta
$$

Note that the real and imaginary parts of (4.18), respectively, give

$$
\begin{aligned}
& X=\xi+\frac{1}{\pi}\left(e^{\pi \xi} \cos \pi \eta+1\right), \\
& Y=\eta+\frac{e^{\pi \xi} \sin \pi \eta}{\pi} .
\end{aligned}
$$

When reaching out to the outer region, $\xi \rightarrow \infty$ (see Figure 6), relations (4.21) yield

$$
\begin{aligned}
& X \sim \frac{e^{\pi \xi} \cos \pi \eta}{\pi}, \\
& Y \sim \frac{e^{\pi \xi} \sin \pi \eta}{\pi},
\end{aligned}
$$

where the relative error is exponentially small. At leading order, we then obtain

$$
\eta \sim \frac{1}{\pi} \arctan \frac{Y}{X}
$$

Reverting to the unscaled coordinates using (3.2b) and substituting into (4.20) again refurnish (4.16). Thus, asymptotic matching with the outer potential (4.5) is trivially satisfied. Similarly, when approaching the gap region $\xi \rightarrow-\infty$ (see Figure 6) whence relations (4.21) become

$$
\begin{array}{r}
X \sim \xi+\frac{1}{\pi}, \\
Y \sim \eta,
\end{array}
$$

again with an exponentially small relative error. From (4.20) and (4.24b), we find that $\varphi \sim Y$, so that asymptotic matching with the gap solution (4.2) is, too, trivially satisfied.

\section{Evaluating the capacitance integral}

Consider now the integral (2.7). Roughly speaking, the gap solution (4.2) applies for $x<1$, while the outer solution (4.5) applies for $x>1$. A naive attempt to evaluate (2.7) using these solutions would result in a logarithmically divergent outer contribution. A similar obstacle was encountered by both Landau and Lifshitz [21] and Binns and Lawrenson [3].

The systematic evaluation of (2.7) makes use of two ingredients. The first is the edge solution (4.12), which applies in the vicinity of $x=1$. The second is the use of a cutoff-like parameter $\delta$, which naturally disappears in the final evaluation of (2.7). Since that parameter is otherwise arbitrary, we choose it at will. Thus, following Hinch [12], we choose it to satisfy the asymptotic condition

$$
\epsilon \ll \delta \ll 1
$$

For example, it may be taken as $\epsilon^{\alpha}$ for any $0<\alpha<1$. 
Using that parameter, we split the capacitance integral (2.7) as follows:

$$
c=\left.\left(\int_{0}^{1-\delta}+\int_{1-\delta}^{1+\delta}+\int_{1+\delta}^{\infty}\right) \frac{\partial \varphi}{\partial y}\right|_{y=0} d x .
$$

The three integrals appearing in (5.2), associated with the contributions of the gap, edge and outer regions, are, respectively, denoted by $c_{g}, c_{e}$ and $c_{o}$. Upon using the natural coordinates in each asymptotic region, we find that the gap contribution is

$$
c_{g}=\left.\frac{1}{\epsilon} \int_{0}^{1-\delta} \frac{\partial \varphi}{\partial Y}\right|_{Y=0} d x
$$

the edge contribution is

$$
c_{e}=\left.\int_{-\delta / \epsilon}^{\delta / \epsilon} \frac{\partial \varphi}{\partial Y}\right|_{Y=0} d X
$$

and the outer contribution is

$$
c_{o}=\left.\int_{1+\delta}^{\infty} \frac{\partial \varphi}{\partial y}\right|_{y=0} d x .
$$

In general, each of these contribution depends both upon $\epsilon$ and $\delta$; their sum, however, cannot depend upon the arbitrary parameter $\delta$, which does not appear in the problem formulation.

The evaluation of the gap and outer contributions is straightforward. Thus, substitution of the gap potential (4.2) into (5.3) gives

$$
c_{g}=\frac{1}{\epsilon}-\frac{\delta}{\epsilon}
$$

with an exponentially small error. In the outer region, we find from (4.5) that

$$
\left.\frac{\partial \varphi}{\partial y}\right|_{y=0}=\frac{2}{\pi\left(x^{2}-1\right)} .
$$

Substitution into (5.5) gives

$$
c_{o} \sim \frac{1}{\pi} \ln \frac{2}{\delta}
$$

where the error is algebraically small (i.e. smaller than some positive power of $\epsilon$ ). The latter expression clearly exhibits the logarithmic divergence of the outer contribution.

In calculating the edge contribution (5.4), we note that both (4.9) and (4.18) map the $\xi$-axis to the $X$-axis. Thus, regardless of which one is employ, we have

$$
d X=f^{\prime}(\xi) d \xi \quad \text { at } \quad \eta=0
$$

Moreover, making use of the manner by which normal derivatives transform under conformal mappings [5], we note that

$$
\left.\frac{\partial \varphi}{\partial Y}\right|_{Y=0}=\left.\frac{\partial \varphi / \partial \eta}{\left|f^{\prime}(\xi)\right|}\right|_{\eta=0}
$$


We therefore find that (5.4) becomes

$$
c_{e}=\left.\int_{f^{-1}(-\delta / \epsilon)}^{f^{-1}(\delta / \epsilon)} \frac{\partial \varphi}{\partial \eta}\right|_{\eta=0} d \xi .
$$

If we employ the Schwarz-Christoffel transformation (4.9), we have, using (4.12),

$$
\left.\frac{\partial \varphi}{\partial \eta}\right|_{\eta=0}=\frac{1}{\pi \xi} .
$$

The numbers $Z= \pm \delta / \epsilon$ are, respectively, associated with points $D$ and $E$ in Figure 5 . Their preimages, $f^{-1}( \pm \delta / \epsilon)$, are associated with points $D^{\prime}$ and $E^{\prime}$. These preimages have positive real part and zero imaginary part. Substitution of (5.12) into (5.11) accordingly gives

$$
c_{e}=\frac{1}{\pi} \ln \frac{f^{-1}(\delta / \epsilon)}{f^{-1}(-\delta / \epsilon)} .
$$

All that remains is to evaluate the preimages $f^{-1}( \pm \delta / \epsilon)$. Since $\delta / \epsilon \gg 1$ (recall (5.1)), it is readily seen from (4.9) that

$$
f^{-1}(\delta / \epsilon) \sim \frac{\pi \delta}{\epsilon},
$$

with a relative error which is algebraically small. Similarly, we find that

$$
\ln f^{-1}(-\delta / \epsilon) \sim-\frac{\pi \delta}{\epsilon}-1
$$

with an algebraically small error. Substitution of (5.14)-(5.15) into (5.13) provides the requisite edge contribution:

$$
c_{e} \sim \frac{1}{\pi} \ln \frac{\pi \delta}{\epsilon}+\frac{\delta}{\epsilon}+\frac{1}{\pi},
$$

with an algebraically small error.

As an alternative, we may employ the mapping (4.18). We then note that (5.9)-(5.10) still hold at $\eta=0$. The edge contribution (5.4) is then still given by (5.11). Here, however, substitution of (4.20) yields (cf. (5.13))

$$
c_{e}=f^{-1}(\delta / \epsilon)-f^{-1}(-\delta / \epsilon) .
$$

The numbers $Z= \pm \delta / \epsilon$ are now, respectively, associated with points $N$ and $P$ in Figure 6 . Their preimages, $f^{-1}( \pm \delta / \epsilon)$, are associated with points $N^{\prime}$ and $P^{\prime}$. All that remains is to evaluate these preimages. Since $\delta / \epsilon \gg 1$ (recall (5.1)), it is readily seen from (4.18) that

$$
f^{-1}(-\delta / \epsilon) \sim-\frac{\delta}{\epsilon}-\frac{1}{\pi}
$$

with a relative error which is algebraically small. In this approximation, the term $e^{\pi \zeta}$ in (4.18) is exponentially small. On the other hand, when seeking $f^{-1}(\delta / \epsilon)$, which is presumably large and negative, it is evident that this term dominates both $\zeta$ and the constant term, suggesting that

$$
f^{-1}(\delta / \epsilon) \sim \frac{1}{\pi} \ln \frac{\pi \delta}{\epsilon} .
$$


Inspection reveals that the leading-order correction to (5.19) is $o(1)$. By substitution of (5.18)(5.19) into (5.17), we obtain (5.16).

Adding up the respective contributions to (5.2), namely (5.6), (5.8) and (5.16), we find that the dependence upon $\delta$ disappears, as it should, giving

$$
c \sim \frac{1}{\epsilon}+\frac{1}{\pi}\left(\ln \frac{2 \pi}{\epsilon}+1\right),
$$

with an algebraically small error. This indeed coincides with the Bromwich-Love approximation (1.2). Thomson's approximation (1.1) misses an $O(1)$ term.

\section{Electric force}

Having calculated the electric potential in the three asymptotic regions, we can exploit the results to calculate the attractive electrostatic force (per unit length) between the two plates. Normalised by $\varepsilon_{0} V^{2} / l$, the force on the upper plate is

$$
\mathbf{F}=\oint\left(\nabla \varphi \nabla \varphi-\frac{1}{2}|\nabla \varphi|^{2} \mid\right) \cdot \hat{\mathbf{n}} d s,
$$

where the notation of (2.5) is used and I denotes the idemfactor. Upon deforming the contour and making use of conditions (2.3)-(2.4), we represent the attractive force $F=-\hat{\mathbf{e}}_{y} \cdot \mathbf{F}$ as an integral over the $x$-axis, namely

$$
F=\frac{1}{2} \int_{-\infty}^{\infty}\left(\frac{\partial \varphi}{\partial y}\right)_{y=0}^{2} d x
$$

or, upon making use of the symmetry about $x=0$,

$$
F=\int_{0}^{\infty}\left(\frac{\partial \varphi}{\partial y}\right)_{y=0}^{2} d x
$$

The idealised approximation (2.8) gives the familiar expression [27, Section 3.7]

$$
F \approx \frac{1}{\epsilon^{2}}
$$

Our goal is the leading-order correction to (6.4).

Making use of the cutoff parameter $\delta$, we decompose $F$ as

$$
F=\left(\int_{0}^{1-\delta}+\int_{1-\delta}^{1+\delta}+\int_{1+\delta}^{\infty}\right)\left(\frac{\partial \varphi}{\partial y}\right)_{y=0}^{2} d x .
$$

The three integrals appearing in the above, respectively, associated with the contributions of the gap, edge and outer regions, are, respectively, denoted by $F_{g}, F_{e}$ and $F_{o}$. Upon using the natural coordinates in each asymptotic region, we find that the gap contribution is

$$
F_{g}=\frac{1}{\epsilon^{2}} \int_{0}^{1-\delta}\left(\frac{\partial \varphi}{\partial Y}\right)_{Y=0}^{2} d x
$$

the edge contribution is

$$
F_{e}=\frac{1}{\epsilon} \int_{-\delta / \epsilon}^{\delta / \epsilon}\left(\frac{\partial \varphi}{\partial Y}\right)_{Y=0}^{2} d X
$$


and the outer contribution is

$$
F_{o}=\int_{1+\delta}^{\infty}\left(\frac{\partial \varphi}{\partial y}\right)_{y=0}^{2} d x .
$$

The evaluation of the gap and outer contributions is straightforward. Thus, substitution of the gap potential (4.2) into (6.6) gives

$$
F_{g}=\frac{1}{\epsilon^{2}}-\frac{\delta}{\epsilon^{2}}
$$

with an exponentially small error. In the outer region, substitution of (5.7) into (6.8) gives

$$
F_{o} \sim \frac{1}{\pi^{2} \delta}
$$

where the error is algebraically small.

In calculating the edge contribution (6.7), we make use of (5.9)-(5.10) to obtain

$$
F_{e}=\frac{1}{\epsilon} \int_{f^{-1}(-\delta / \epsilon)}^{f^{-1}(\delta / \epsilon)} \frac{1}{f^{\prime}(\xi)}\left(\frac{\partial \varphi}{\partial \eta}\right)_{\eta=0}^{2} d \xi .
$$

Substituting (4.9) and (5.12) gives

$$
F_{e}=\frac{1}{\pi \epsilon}\left[\ln \frac{f^{-1}(\delta / \epsilon)}{f^{-1}(\delta / \epsilon)+1}-\ln \frac{f^{-1}(-\delta / \epsilon)}{f^{-1}(-\delta / \epsilon)+1}\right] .
$$

While approximation (5.15) is sufficient for our needs, we need a refinement of (5.14). It is not difficult to see from (4.9) that

$$
f^{-1}(\delta / \epsilon) \sim \frac{\pi \delta}{\epsilon}-\left(\ln \frac{\pi \delta}{\epsilon}+1\right),
$$

with a relative error which is algebraically small. Substitution into (6.12) provides the requisite edge contribution:

$$
F_{e} \sim-\frac{1}{\pi^{2} \delta}+\frac{\delta}{\epsilon^{2}}+\frac{1}{\pi \epsilon}
$$

with an algebraically small error.

Alternatively, we could use (4.18) and (4.20) instead of the Schwarz-Christoffel transformation (4.9). Substitution into (6.14) then gives, instead of (6.12),

$$
F_{e}=\frac{1}{\pi \epsilon}\left[\xi-\frac{\ln \left(1+e^{\pi \xi}\right)}{\pi}\right]_{f^{-1}(-\delta / \epsilon)}^{f^{-1}(\delta / \epsilon)} .
$$

Making use of (5.18)-(5.19), we retrieve (6.14).

Adding up the respective contributions to (6.5), namely (6.9), (6.10) and (6.14), we find that the dependence upon $\delta$ disappears, giving

$$
F \sim \frac{1}{\epsilon^{2}}+\frac{1}{\pi \epsilon},
$$

with an algebraically small error. The ratio of the above approximation to the idealised capacitor formula (6.4) is $1+\epsilon / \pi$. 
An indirect derivation of (6.16), based upon the principle of virtual work and the capacitance approximation (5.20), is presented in the Appendix.

\section{Concluding remarks}

Making use of matched asymptotic expansions, we have analysed the electrostatics of a parallelplate capacitor in the limit of small separation. Our analysis has reconfirmed the result of Bromwich [4] and others, thus showing that the Thomson's approximation for capacitance edge correction misses a term. We have additionally calculated the electrostatic attraction between the plates. Measurement of that force has been used for the determination of $\varepsilon_{0}$, the permittivity of free space $[11,32]$. The refined approximation (6.16) for the dependence of the force upon the separation between the plates may yield a more accurate determination.

There is some resemblance between the present analysis and that of Jeffrey [17], where a two-sphere capacitor was considered. There are two main technical differences between the two problems. The first is obvious: in Jeffrey's analysis, there are two distinct asymptotic regions, namely, the gap and outer regions; in the present problem, one needs to distinguish between three regions.

The second difference has to do with asymptotic corrections. In both Ref. [17] and the present analysis, the parameter $\epsilon$ appears only in the gap region, due to the non-uniform manner by which the $x$ and $y$ coordinates are stretched (see (4.1)). In Ref. [17], where the gap is analysed using a lubrication approximation, the presence of the small parameter in the differential equation naturally leads to higher order corrections. This is not the case here, where the gap solution (4.2) satisfies the exact equation (4.1), and the error is exponentially small. Since the errors associated with the leading-order solutions in the outer and edge regions are not exponentially small, higher order corrections emerge in these regions as a consequence of higher order asymptotic matching [28]. In the present contribution, where we sought the leading-order correction to the idealised capacitor approximations, no need arises for the calculation of such higher order terms.

\section{Conflicts of interest}

None.

\section{References}

[1] Atkinson, C. \& Leppington, F. G. (1983) The asymptotic solution of some integral equations. IMA J. Appl. Math. 31, 169-182.

[2] AtKinson, C. \& SHeRwood, J. D. (2017) Added mass of a pair of disks at small separation. Eur. J. Appl. Math. 28, 687-706.

[3] Binns, K. J. \& Lawrenson, P. J. (1973) The Analytical and Numerical Solution of Electric and Magnetic Field Problems, Pergamon Press, New York.

[4] Bromwich, T. J. I'A. Note on condenser problems. Mess. Math. 31, 184 (1902).

[5] Brown, J. W. \& Churchill, R. V. (2003) Complex Variables and Applications, McGraw-Hill, New York.

[6] Carlson, G. T. \& Illman, B. L. (1994) The circular disk parallel plate capacitor. Am. J. Phys. 62, 1099-1105. 
[7] Chew, W. C. \& Kong, J. A. (1981) Asymptotic formula for the capacitance of two oppositely charged discs. Math. Proc. Cambridge Philos. Soc. 89, 373-384.

[8] Cooke, J. C. (1958) The coaxial circular disc problem. ZAMM 38, 349-356.

[9] Feynman, R. P., Leighton, R. B. \& Sands, M. (1979) The Feynman Lectures on Physics, Vol. 2: Mainly Electromagnetism and Matter, Addison-Wesley, Massachusetts.

[10] Grove, T. T., Masters, M. F. \& Miers, R. E. (2005) Determining dielectric constants using a parallel plate capacitor. Am. J. Phys. 73, 52-56.

[11] Harwood, R. J. \& KinKaid, D. E. (1975) Use of a Mettler balance and a parallel plate capacitor to measure the permittivity of free space. Am. J. Phys. 43, 924-925.

[12] Hinch, E. J. (1991) Perturbation Methods, Cambridge University Press, Cambridge.

[13] Hutson, V. (1963) The circular plate condenser at small separations. Math. Proc. Cambridge Philos. Soc. 59, 211-224.

[14] Ignatowsky, W. (1932) Kreisscheibenkondensator. Akad. Sci. U.R.S.S. Trav. Inst. Stekloff. 3, $1-104$.

[15] JACKSON, J. D. (1999) A curious and useful theorem in two-dimensional electrostatics. Am. J. Phys. 67, 107-115.

[16] JACKSON, J. D. (2002) Charge density on a thin straight wire: the first visit. Am. J. Phys. 70, 409-410.

[17] JeFFREY, D. J. (1978) The temperature field or electric potential around two almost touching spheres. J. Inst. Math. Appl. 22, 337-351.

[18] In Ref. [17], the latter is denoted "inner".

[19] Kirchhoff, G. R. (1877) Zur theorie des condensators. Monatsb. Deutsch. Acad. Wiss. Berlin, $144-172$.

[20] Kromhout, R. A. \& Moulton, W. G. (1956) Effect of fringing field on capacitance and a measurement of $\varepsilon_{0}$. Am. J. Phys. 24, 631-632.

[21] Landau, L. D. \& Lifshitz, E. M. (1960) Course of Theoretical Physics: Electrodynamics of Continuous Media, Permagon Press.

[22] Leppington, F. \& Levine, H. (1970) On the capacity of the circular disc condenser at small separation. Math. Proc. Cambridge Philos. Soc. 68, 235-254.

[23] Love, A. E. H. (1924) Some electrostatic distributions in two dimensions. P. Lond. Math. Soc. 2 , 337-369.

[24] NAINI, A. \& Green, M. (1977) Fringing fields in a parallel-plate capacitor. Am. J. Phys. 45, 877-879.

[25] Nishiy ama, H. \& NAKAmura, M. (1990) Capacitance of a strip capacitor. IEEE Trans. Compon. Hybrids Manuf. Technol. 13, 417-423.

[26] Parker, G. W. (2002) Electric field outside a parallel plate capacitor. Am. J. Phys. 70, 502-507.

[27] Purcell, E. M. (1985) Electricity and Magnetism, Berkeley Physics Course, Vol. 2, McGraw-Hill.

[28] SHAw, S. J. N. (1970) Circular-disk viscometer and related electrostatic problems. Phys. Fluids 13, $1935-14$.

[29] Sneddon, I. N. (1966) Mixed Boundary Value Problems in Potential Theory, Wiley, New York).

[30] Thomson, J. J. (1893) Recent Researches in Electricity and Magnetism, Clarendon, Oxford, UK, pp. 208-250.

[31] Wintle, H. J. \& Kurylowicz, S. (1985) Edge corrections for strip and disc capacitors. IEEE Trans. Instrum. Meas. 34, 41-47.

[32] YAn, F. N. \& Wong, H. K. (1993) Force between the plates of a parallel-plate capacitor. Am. J. Phys. 61, 1153-1153.

\section{Appendix A Derivation of (6.16) using the principle of virtual work}

Going back to dimensional notation, the energy (per unit length) of the capacitor is

$$
U=\frac{Q^{2}}{2 C}
$$


By considering a virtual displacement, with the plates conceptually disconnected from any voltage source. With the plate separation $2 h$ undergoing an infinitesimal change $2 d h$, the external force required to hold the plates in place is

$$
\frac{1}{2} \frac{d}{d h}\left(\frac{Q^{2}}{2 C}\right)
$$

This is then also the expression for the electric force (per unit length), where a positive sign corresponds to an attractive electric force. (This is a natural generalisation of expression (6.3), which provides the force on the upper plate.) Since the charge $Q$ is unaffected by the above virtual displacement, it follows that [27]

$$
\text { the electric force (per unit length) }=-\frac{Q^{2}}{4 C^{2}} \frac{d C}{d h},
$$

or, using (2.1),

$$
\text { the electric force (per unit length) }=-V^{2} \frac{d C}{d h} .
$$

Now, the dimensional version of (5.20) is

$$
C \approx \varepsilon_{0}\left[\frac{l}{h}+\frac{1}{\pi}\left(\ln \frac{2 \pi l}{h}+1\right)\right],
$$

so the corresponding approximation to the force is

$$
\text { the electric force (per unit length) } \approx \frac{\varepsilon_{0} V^{2}}{l}\left(\frac{l^{2}}{h^{2}}+\frac{l}{\pi h}\right) .
$$

The associated dimensionless force coincides with (6.16). 